\title{
Pharmacists' interventions on clinical asthma outcomes: a systematic review
}

\author{
Victoria Garcia-Cardenas ${ }^{1}$, Carol Armour ${ }^{2}$, Shalom I. Benrimoj ${ }^{1}$, \\ Fernando Martinez-Martinez ${ }^{3}$, Inajara Rotta ${ }^{4}$ and Fernando Fernandez-Llimos ${ }^{5}$
}

\author{
Affiliations: \\ ${ }^{1}$ Graduate School of Health, University of Technology Sydney, Sydney, Australia. \\ ${ }^{2}$ Woolcock Institute of Medical Research, The University of Sydney, Sydney Local Health District, Sydney, \\ Australia. \\ ${ }^{3}$ Pharmaceutical Care Research Group, University of Granada, Granada, Spain. \\ ${ }^{4}$ Hospital Pharmacy Dept, Hospital de Clínicas, Federal University of Paraná, Curitiba, Brazil. \\ ${ }^{5}$ Research Institute for Medicines (iMed. ULisboa), Dept of Social Pharmacy, Faculty of Pharmacy, University \\ of Lisbon, Lisbon, Portugal.
}

\section{Correspondence:}

Victoria Garcia-Cardenas, Graduate School of Health, University of Technology Sydney, City Campus, Broadway, Building 7, Level 4, Room 41, PO Box 123, Broadway, NSW 2007, Australia.

E-mail: Victoria.GarciaCardenasQuts.edu.au

ABSTRACT The objective of this systematic review was to evaluate the impact of pharmacists' interventions on clinical asthma outcomes on adult patients and to identify the outcome indicators used.

PubMed, Scopus, Web of Science and Scielo were searched. Studies addressing pharmacists' interventions on adult asthma patients reporting clinical asthma outcomes were incorporated.

11 clinical outcomes were identified in 21 studies. 10 studies measured the impact of the intervention on asthma control. Randomised controlled trials (RCT) and non-RCTs found positive results in percentages of controlled patients and Asthma Control Questionnaire (ACQ) scores. Discordant results were found for Asthma Control Test results. Asthma severity was assessed in four studies. One RCT found a significant decrease in the percentage of severe patients; two non-RCTs found significant improvements in severity scores. 11 studies reported pulmonary function indicators, showing inconsistent results. Eight studies measured asthma symptoms; three RCTs and four non-RCTs showed significant improvements.

RCTs and non-RCTs generated similar results for most outcomes. Based on the evidence generated by RCTs, pharmacists' have a positive impact on the percentage of controlled patients, ACQ scores, severity and symptoms. Future research should report using the core outcome set of indicators established for asthma (PROSPERO CRD42014007019).

@ERSpublications

This systematic review finds that pharmacists have a positive impact on various clinical asthma outcomes http://ow.ly/UCjin

For editorial comment see Eur Respir J 2016; 47: 1043-1046 [DOI: 10.1183/13993003.00240-2016].

This article has supplementary material available from erj.ersjournals.com

Received: Aug 252015 | Accepted after revision: Nov 082015 | First published online: Dec 172015

Conflict of interest: None declared.

Copyright OERS 2016 


\section{Introduction}

According to World Health Organization estimates [1], 235 million people worldwide suffer from asthma, making it a major health problem in industrialised countries. The social impact of asthma is high, with negative clinical, economic and humanistic implications [2], mainly due to ineffective management of the disease.

Good asthma outcomes hinge on the accessibility of effective medications and their appropriate use by patients. Regular reviews with a healthcare provider are an essential component for effective asthma management [3]. Asthma education and training can be delivered effectively by different healthcare providers, such as physicians, nurses or pharmacists. Given that many of the issues associated with suboptimal asthma management are related to the inappropriate use of medications $[4,5]$, pharmacists are in an excellent position to play an active and positive role in the management of asthma. The change in pharmacists' practice in healthcare to a more patient-centred approach, through the provision of professional pharmacy services, supports and focuses on optimising the use of medicines and improving health outcomes. Several meta-analyses have shown a positive impact of pharmacists when delivering clinical services for patients with chronic conditions such as diabetes [6] or hypertension [7]. Similarly, a literature review found that community pharmacists can play an effective role in screening for poorly controlled asthma and undiagnosed chronic obstructive pulmonary disease by delivering management interventions [8]. A narrative review revealed an expanding role in asthma care across different settings [9]. However, no systematic review of pharmacists' impact on asthma outcomes has been found.

Selecting appropriate outcomes when designing any research study is crucial when reporting the results of the research, since it allows analysing the effects of different interventions in ways that minimise bias [10]. However, there seems to be a high variability in the literature when reporting the effects of interventions on asthma patients. Difficulties caused by the heterogeneity of outcome measurements are common. This heterogeneity has direct implications when comparing and analysing the evidence available. However, this problem could be addressed by the design of a "core outcome set", which is an agreed minimum set of outcomes or outcome measures (The COMET (Core Outcome Measures in Effectiveness Trials) Initiative: www.comet-initiative.org) [11]. This implies a standardisation of the variables that should be measured and reported in all trials in a specific area. Although there is a growing recognition of its relevance and some work has already been undertaken in childhood asthma, to the best of our knowledge no core outcome set has been established for adult asthma patients in community care.

The objective of this systematic review was to evaluate the impact of pharmacists' interventions on clinical asthma outcomes on adult patients and to identify the clinical outcome indicators reported in experimental studies to assess them.

\section{Material and methods}

A systematic review was undertaken following the methodological and reporting standards recommended by PRISMA [12] and AMSTAR [13]. A literature search was conducted in August 2015. Neither publication date nor publication type filters were used. Studies assessing pharmacists' interventions on adult asthma patients reporting clinical asthma outcomes as a result of the intervention provided were included. The studies eligible were those published or at least with an abstract and written with the Latin alphabet. Searches were conducted in PubMed, Scopus, Web of Science and Scielo. The queries used are described in table 1. Duplicates records were removed.

The literature selection process was undertaken and discussed between two experts on asthma services (F.F.-L. and V.G.-C.). To identify potentially relevant articles, a screening of records retrieved from the search was performed by reviewing titles and abstracts. This process was over-inclusive. Obviously irrelevant records were removed. Potentially relevant articles were retrieved and multiple reports of the same study were linked together. Full-text papers not discarded in the screening were read and studies were excluded according to the following criteria: 1) nonexperimental studies, 2) studies with asthma patients younger than 18 years old, 3) studies not reporting a clinical asthma outcome (e.g. asthma control, asthma severity, asthma symptoms, peak expiratory flow (PEF), forced expiratory volume in $1 \mathrm{~s}$ (FEV1) or other pulmonary function indicator) and 4) studies in which the educational intervention was not exclusively provided by a pharmacist. The reference lists of the retrieved papers were reviewed for potentially additional relevant studies.

Relevant information from all included studies was gathered using a pre-designed and piloted data extraction form. The following information was extracted. 1) Source: study ID, citation and contact details. 2) Eligibility (confirmation of inclusion criteria). 3) Objective. 4) Methods: study design, study groups, follow-up time, sequence generation, allocation sequence concealment, blinding. 5) Participants: total number of patients, total number of practices, setting, country, inclusion/exclusion criteria. 6) Interventions: number of intervention groups, intervention details, outcomes, outcome definitions, method of assessment, 


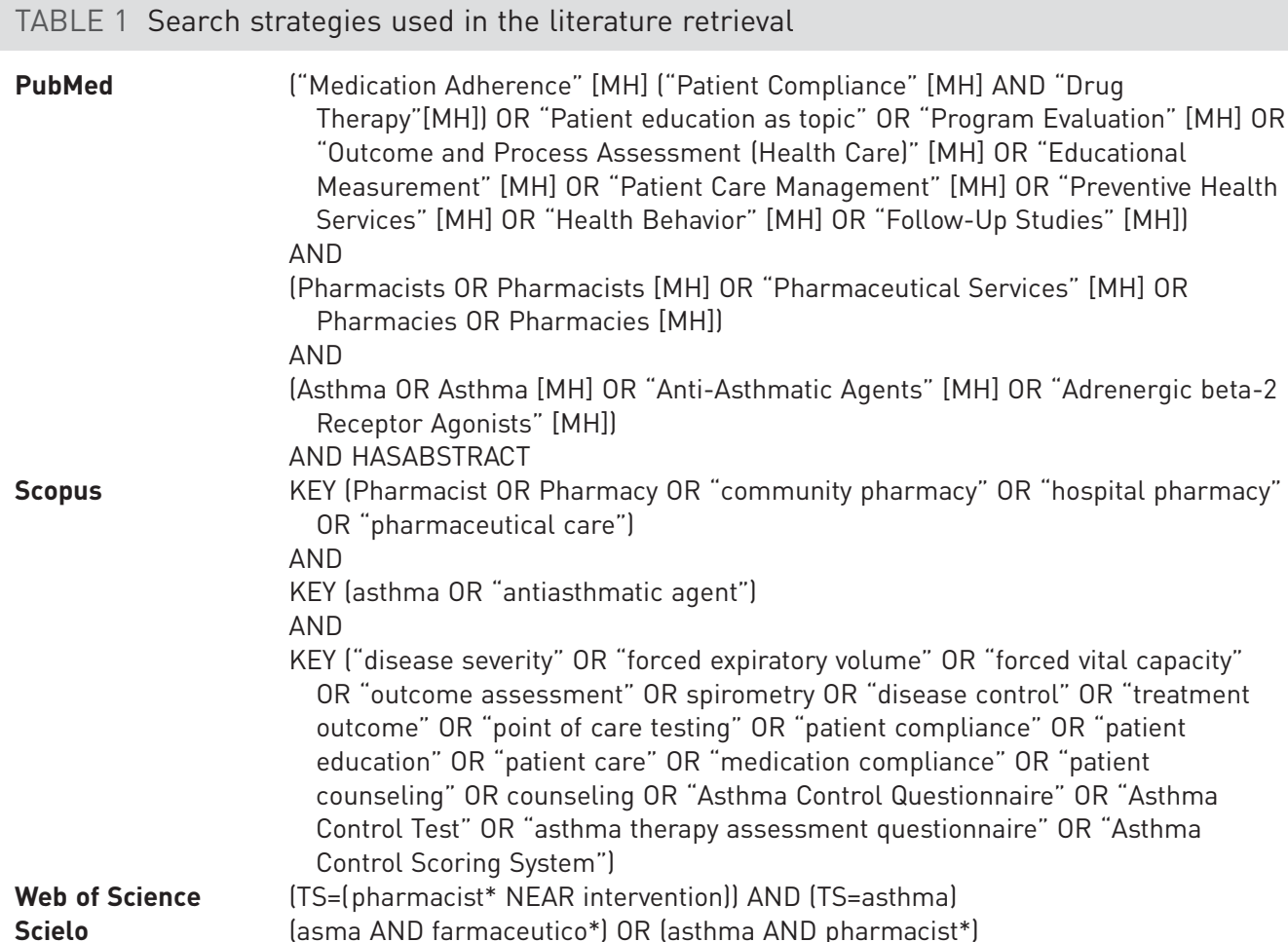

characteristics of the method of assessment (if ad hoc). 7) Results: Number of patients and practices allocated to each group, sample size, missing participants, subgroup analysis. 8) Summary of data: summary of results for each clinical asthma outcome assessed. 9) Conclusions. 10) Miscellaneous: funding source, references to other relevant studies, reviewer's comments.

Following AMSTAR recommendations, data synthesis and conclusions were formulated taking into consideration the epidemiological design of the studies [13]. The systematic review was registered in the PROSPERO international prospective register of systematic reviews database (registration CRD42014007019), where a detailed protocol of the review can be found.

\section{Results}

Initially, 1194 different potential articles were retrieved from the databases used. After screening by title and abstract, 68 of them were selected for full-text review. In the second step, 44 were excluded for the following reasons (papers could be excluded due to more than one criterion): six were nonexperimental studies, 22 included a population under 18 years old, in 10 the intervention was not delivered exclusively by a pharmacist and 19 did not evaluate any clinical asthma outcome. 24 papers corresponding to 21 studies were included (figure 1). 14 studies were conducted in a community pharmacy setting [14-27], two in a hospital setting $[28,29]$, one in a community pharmacy setting in collaboration with physician practices [30], one in a community clinic setting [31], one in an outpatient medical centre [32], one in an antenatal outpatient clinic [33] and one was conducted by telephone [34]. Seven studies were conducted under a randomised controlled trial (RCT) design [16, 17, 22, 28, 29, 33, 34], two under a cluster RCT (C-RCT) design [14, 19], two under a cluster randomised trial design [15, 26], two under a cluster controlled trial design $[24,25]$ and eight under a quasi-experimental study with no control group design $[18,20,21,23,27,30-32]$. The follow-up period varied across the studies, ranging between $1[20,26], 2$ [28], $3[16], 5[18,29], 6[14,15,17,19,22,23,27,31,33,34], 9[25], 12[21,24]$ and 24 months [30]. The follow-up period was not specified in one of the studies retrieved [32]. The main characteristics of the studies are summarised in the online supplementary material.

\section{Impact of pharmacists' interventions on clinical asthma outcomes}

\section{Current asthma control}

10 studies measured the impact of pharmacists' interventions on current asthma control as a main outcome $[15,17,19,20,22,25-27,33,34]$. It was mainly assessed using validated instruments, such as the 

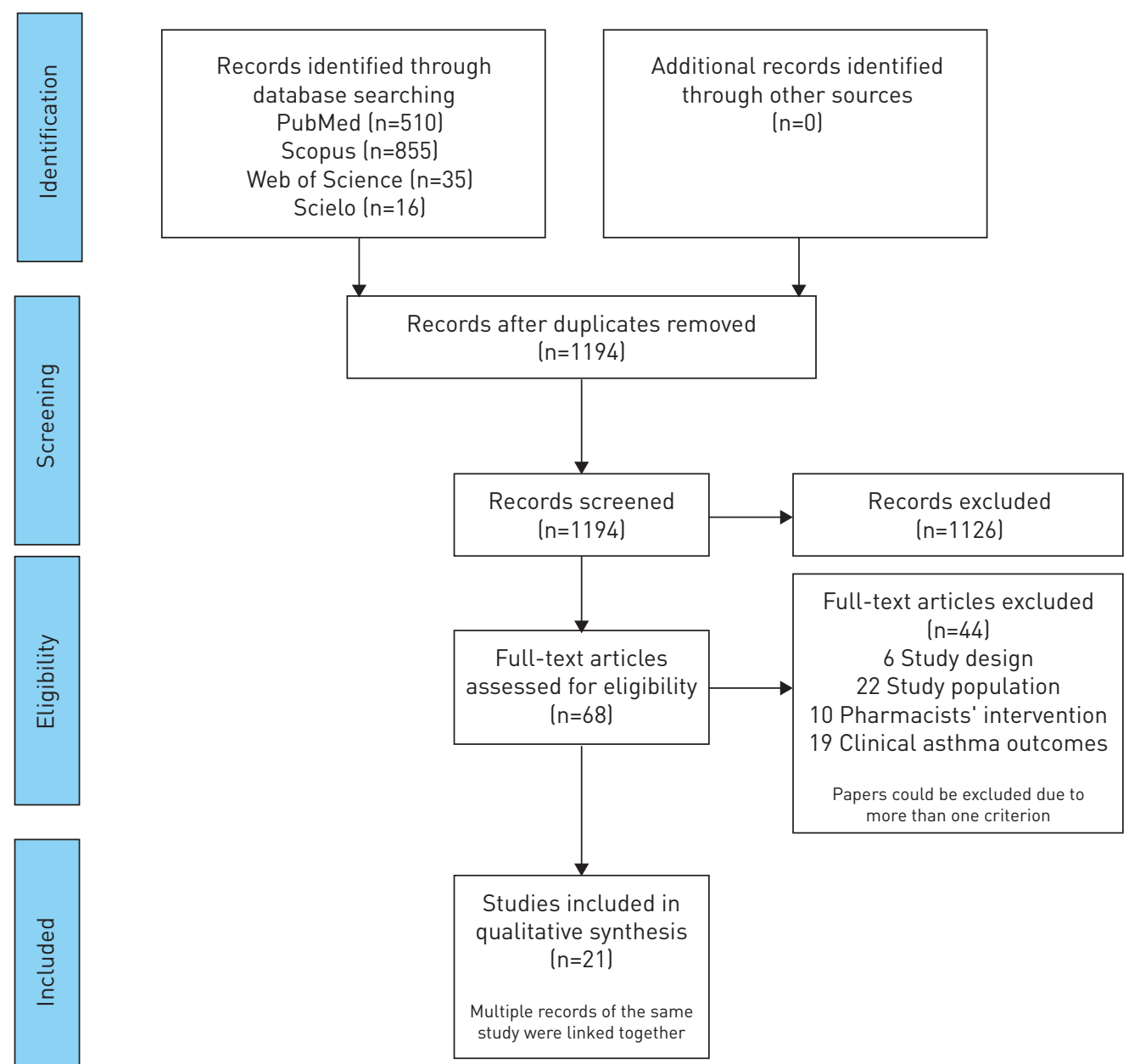

FIGURE 1 Flow diagram of studies included in the review.

Asthma Control Questionnaire (ACQ) and the Asthma Control Test (ACT). Four studies reported a change in the number of controlled asthma patients $[15,19,22,27]$ and 10 studies reported a change in either ACQ $[15,19,20,25,26,33]$ or ACT $[17,22,27,34]$ scores.

Five studies that measured current asthma control as a main outcome used either a RCT or a C-RCT design $[17,19,22,33,34]$. The two (C)-RCTs that measured the change in the number of controlled asthma patients reported an improvement in the percentage of patients considered to have good asthma control $[19,22]$. Results showed that the difference in the percentage of controlled patients between study groups after the provision of the intervention was $12.1 \%(\mathrm{p}=0.028)$ and $7.7 \%$ (no $\mathrm{p}$-value provided), respectively. One of the studies found that patients in the intervention group were almost three times more likely to have their asthma controlled than patients in the control group (OR 3.06, 95\% CI 1.63-5.73; $\mathrm{p}<0.001)$. The two $(\mathrm{C})$-RCTs assessing the change in ACQ scores also found a positive impact, since both statistically and clinically significant reductions in patient's ACQ scores were observed [19, 33]. One study found a difference in ACQ scores between study groups after the provision of the intervention of 0.41 points $(p<0.001)$ [19], whereas in another the difference was equal to $0.60(p<0.001)$ [33]. Only one of the three RCTs evaluating the change in ACT scores found significant improvements in the intergroup comparisons [17]. This study found a difference in ACT scores between study groups after the intervention equal to $2.6(\mathrm{p}<0.01)$.

The other five studies that measured current asthma control used different research designs [15, 20, 25-27]. Two of them assessed the change in the number of controlled patients, signalling positive findings [15, 27]. One study assessed the impact of two different interventions, reporting a significant improvement in the percentage of patients considered to have good asthma control after both interventions were delivered (increase of $32 \%$ and $38 \%$, respectively; no p-value provided for the intra-group comparisons) [15]. Four studies reported the change in ACQ scores $[15,20,25,26]$. These four found significant intra-group 
reductions in ACQ scores of $0.56-0.57$ (no $p$-value provided), $0.4(p<0.001), 0.23(p=0.02)$ and 0.4 $(\mathrm{p}=0.003)$, respectively. One study reported statistical and clinical significant reductions, since $48 \%$ of patients demonstrated a reduction $\geqslant 0.5$ in their ACQ scores [15].

Asthma severity

The impact of pharmacy interventions on asthma severity was assessed in four studies [14, 21, 23, 24]. The methods of assessment varied: two studies used the criteria established by the German Asthma Guidelines [21, 24], one used a tool based on the National Asthma Council Australia severity assessment table [14], whereas another based the assessment on the criteria established by the Australian Asthma Management Handbook [23]. In terms of the impact of the pharmacists' interventions, one study reported the change in the number of patients suffering from severe asthma [14] and three studies used the change in mean asthma severity scores as their outcome indicator [21, 23, 24].

The only RCT assessing the impact of pharmacists' interventions on asthma severity reported a significant decrease in the percentage of patients having severe asthma [14]. This study reported a significant decrease in the proportion of severe patients from $87.9 \%$ to $52.7 \%(\mathrm{p}<0.001)$, while the control group remained unchanged. The authors also found that patients in the intervention group were more likely to change from the severe to the not severe category than patients in the control group (OR 2.68, 95\% CI 1.64-4.37; $\mathrm{p}<0.001$ ).

Three further studies assessing asthma severity as a main outcome indicator used a nonrandomised design. Two studies reported significant decreases in mean asthma severity scores $[21,23](-0.3, \mathrm{p}<0.002$ and $-0.3, \mathrm{p}<0.001$, respectively) and one did not find any significant difference [24].

\section{Pulmonary function}

11 studies reported some measurement of pulmonary function as an outcome indicator of the intervention designed [14, 18, 21-24, 27-30, 32]. Seven reported changes in PEF values [18, 21-24, 29, 30], six in FEV 1 values $[14,21,24,27,28,32]$, one in vital capacity (VC) [21], one in FEV1 \% VC (FEV1 expressed as a percentage of the VC, Tiffeneau index) [21], one in forced vital capacity (FVC) [32] and one in percentage of FEV1/FVC predicted values [14]. Methods of evaluation and reporting of outcome indicators diverged. PEF values were either measured at the pharmacy $[18,21,24,29,30]$ or self-measured by the patient [22$24]$. Results were expressed as PEF rate [18, 21, 24, 29], percentage of maximum predicted PEF [22], peak flow index [23], and number of patients below $70 \%$ and $85 \%$ of optimal PEF [30]. In terms of FEV 1 values, they were measured by a pharmacist [14] or by a physician [21]. This information was unknown in four studies $[24,27,28,32]$. Results were reported as percentage predicted [14, 27], as an absolute number $[21,28]$, as a mean percentage change [32], as a percentage change from baseline [24] and as a percentage of the VC [21]. The study assessing VC reported it in absolute values [21], whereas the study measuring FVC reported it as mean percentage change [32]. The only study measuring FEV1/FVC reported it as a percentage of predicted value [14].

Four out of the 10 studies evaluating any pulmonary function outcome indicator used a RCT design $[14,22,28,29]$. No studies reported improvements in percentage of maximum PEF [22] or PEF rate [29]. However, one RCT did report significant improvements in FEV1 values after the intervention [28] (difference of $0.20 \mathrm{~L}$ between study groups; no p-value provided). One C-RCT evaluated the change in percentage predicted FEV1/FVC, with no change after the follow-up [14].

Results from non-RCTs showed two studies reporting improvements in PEF rates [18, 21]. In the first study, PEF rate improvements from 0.13 to $0.12 \mathrm{~L} \cdot \mathrm{min}^{-1}$ (no $\mathrm{p}$-value provided) were found. In the second study, an improvement of $0.35 \mathrm{~L} \cdot \mathrm{min}^{-1}(\mathrm{p}<0.001)$ was reported. Only one study was identified as using peak flow index as an outcome indicator [23], with results showing significant improvements after the intervention (from $82.7 \pm 8.2 \%$ to $87.4 \pm 8.9 \%$; $<<0.001$ ). In terms of other outcomes, one study showed significant improvements in the percentage of predicted FEV1 (from $46.6 \pm 0.09 \%$ to $70.4 \pm 0.10 \%$; $p<0.05$ ) [27]. One study reported significant improvements of mean percentage changes in FEV 1 values $(18.5 \pm 1.5 \mathrm{~L}$ in the intervention group versus $5.2 \pm 1.0 \mathrm{~L}$ in the comparison group; no p-value provided), but no differences in FVC [32]. Two studies did not find any effect on other pulmonary function indicators such as VC, FVC or FEV1 \% VC $[21,32]$

\section{Asthma symptoms}

Eight studies reported the impact of pharmacists' interventions on asthma symptoms. Three of them assessed the occurrence of general asthma symptoms [16, 21, 30], evaluated with the validated North England Asthma Symptoms Scale, with a self-reported measure and with an ad hoc questionnaire. This outcome was reported as a mean symptoms score [16], as a self-scored punctuation [21] and as a mean number of symptoms suffered in the previous month [30]. Four studies assessed the occurrence of nocturnal asthma symptoms as outcome indicators, including mean nocturnal episodes of asthma [31], 
mean frequency of nocturnal asthma symptoms [29], number of nocturnal awakenings due to asthma [22] and sleep disturbances [18]. Two studies used a self-reported card/diary to assess asthma nocturnal episodes [22, 29], whereas the method of assessment was not specified in two studies $[18,31]$. Two studies assessed dyspnoea severity $[21,24]$, rated by the patient's physician through the Medical Research Council dyspnoea scale.

Amongst the three RCTs [16, 22, 29], one showed a significant improvement in the mean asthma symptoms score [16], with a mean difference in asthma symptoms scores between study groups after the intervention equal to 7 (95\% CI 4.4-9.5; $\mathrm{p}<0.001$ ). Two studies reported positive outcomes in terms of nocturnal asthma symptoms $[22,29]$; one study reported a difference in mean change of nocturnal awakenings between study groups equal to -3.5 (95\% CI $-7.0--0.1 ; \mathrm{p}=0.004)$, and the second study found that patients in the intervention group had a greater significant decrease in the mean frequency of nocturnal symptoms than patients in the control group after 20 and 22 weeks of follow-up (no mean values provided; $\mathrm{p}<0.05$ ).

Of the five nonrandomised studies assessing the impact on asthma symptoms, one study found a reduction in the number of patients with moderate or severe symptoms [30] (no p-value provided). Three studies reported positive findings in terms of nocturnal asthma symptoms $[18,31]$ and general asthma symptoms [21], and one in terms of dyspnoea severity scores [21].

Table 2 shows a summary of the pharmacists' impact on the different asthma outcomes identified, and the heterogeneity in both the outcomes assessed and the method of reporting.

\section{Meta-analysis}

The use of statistical techniques was attempted to integrate and summarise the results reported for clinical asthma outcomes. However, a meta-analysis could not be performed due to the lack of some statistical

TABLE 2 Summary of findings of the impact of pharmacists' interventions on asthma outcomes

Outcome

Reporting method
Findings in (C)-RCTs
Findings in other research designs

$(+)[15,27]^{\#}$

$(+)[19,22]$

$(+)[17] ;( \pm)[22,34]^{\#}$

$(+)[14]$

NEA

Asthma severity score
Percentage severe patients

PEF rate

Percentage of maximum predicted PEF Peak flow index

PEF values $<85 \%$ of optimal PEF

PEF values $<70 \%$ of optimal PEF

FEV1

VC

FVC

FEV $1 / F V C$

$\mathrm{FEV} 1 \% \mathrm{VC}$

\section{Asthma symptoms}

General symptoms

Nocturnal symptoms

Dyspnoea
Percentage of predicted personal best

FEV1 (absolute number)

Mean percentage change

Percentage change of FEV 1 from baseline Absolute value

Mean percentage change

Percentage of $\mathrm{FEV}_{1} / \mathrm{FVC}$ predicted value

FEV1 expressed as a percentage of the VC (Tiffeneau index)

Mean asthma symptoms score

Self rated score

Mean number of symptoms in previous week

Mean nocturnal episodes of asthma

Mean frequency of nocturnal asthma symptoms

Number of nocturnal awakenings due to asthma

Number of patients with sleep disturbances

Dyspnoea severity score

$\begin{array}{cc}( \pm)[29] & (+)[18,21] ;( \pm)[24] \\ ( \pm)[22] & N E A \\ N E A & (+)[23] \\ N E A & (+)[30]^{\#} \\ N E A & (+)[30]^{\#} \\ ( \pm)[14] & (+)[27] \\ (+)[28]^{\#} & (+)[21]^{\#} \\ N E A & (+)[32] \\ N E A & ( \pm)[24] \\ \text { NEA } & ( \pm)[21] \\ \text { NEA } & ( \pm)[32]^{\#} \\ ( \pm)[14] & N E A \\ \text { NEA } & ( \pm)[21] \\ & \\ (+)[16] & N E A \\ \text { NEA } & (+)[21] \\ \text { NEA } & (+)[30]^{\#} \\ \text { NEA } & (+)[31] \\ (+)[29] & N E A \\ (+)[22] & N E A \\ \text { NEA } & (+)[18]^{\#} \\ \text { NEA } & (+)[21] ;( \pm)[24]\end{array}$

PEF: peak expiratory flow; FEV1: forced expiratory volume in $1 \mathrm{~s}$; VC: vital capacity; FVC: forced vital capacity; ACQ: Asthma Control Questionnaire; ACT: Asthma Control Test; (+): positive findings; ( \pm ): neutral findings. (C)-RCT: (cluster) randomised controlled trial; NEA: no evidence available. ${ }^{\#}$ : no $\mathrm{p}$-value provided. 
TABLE 3 Individual assessment of each study regarding suitability for inclusion in meta-analysis

Outcome assessed Study Justification

\section{Continuous outcomes}

ACQ scores

GaRcia-CARDEnAs et al. [19]

Armour et al. [15]

GIRAUD et al. [20]

SMITH et al. [25]

Lim et al. [33]

ACT scores

Toumas-Sehata et al. [26]

MenuYs et al. [22]

Young et al. [34]

BerEZNICKI et al. [17]

Asthma severity

PEF

FEV1

VC

FVC

$\mathrm{FEV}_{1} / \mathrm{FVC}$

FEV $1 \%$ VC

Asthma symptoms
MANGiaPAne et al. [21]

SAINI et al. [23]

Schulz et al. [24]

MANGIAPANE et al. [21]

SAINI et al. [23]

Menurs et al. [22]

AbDelhamid et al. [29]

Petkova [18]

Schulz et al. [24]

NARHI et al. [30]

MAngIAPANe et al. [21]

AnJAN Kumar et al. [28]

Schulz et al. [24]

Armour et al. [14]

de TulLio and Corson [32]

ZANGHELINI et al. [27]

MANGiaPANe et al. [21]

DE TULLIO and CORSON [32]

Armour et al. [14]

Mangiapane et al. [21]

BARBANEL et al. [16]

Mehurs et al. [22]

Petkova [18]

NARHI et al. [30]

Odegard et al. [31]

Abdelhamid et al. [29]

MAngiapane et al. [21]

Schulz et al. [24]

Menuys et al. [22]

Armour et al. [15]

Garcia-CARDEnAs et al. [19]

ZANGHELINI et al. [27]

Armour et al. [14]
Lack of standard deviation of mean change for control group

Lack of control group

Lack of control group

Lack of standard deviation of mean change for both study groups Appropriate for meta-analysis Lack of control group

Appropriate for meta-analysis

Lack of standard deviation of mean difference

Lack of baseline results for control group; lack of mean change and standard deviation of both study groups Lack of control group

Lack of standard deviation of mean for both groups

Lack of standard deviation of mean for both groups Lack of control group

PEF values only in intervention group Appropriate for meta-analysis

Lack of outcome values before and after in both study groups Lack of control group

Lack of standard deviation of mean change for both study groups Lack of control group Lack of control group

Lack of standard deviation of mean change for both study groups Appropriate for meta-analysis Appropriate for meta-analysis

Lack of standard deviation of mean change and $\mathrm{p}$-value for both study groups Lack of control group Appropriate for meta-analysis Results not reported Appropriate for meta-analysis Appropriate for meta-analysis Appropriate for meta-analysis Appropriate for meta-analysis Lack of control group Lack of control group Lack of control group Appropriate for meta-analysis Lack of control group

Lack of standard deviation of mean change for both study groups

Appropriate for meta-analysis Appropriate for meta-analysis Appropriate for meta-analysis Lack of control group Appropriate for meta-analysis

Asthma severity

ACQ: Asthma Control Questionnaire; ACT: Asthma Control Test; PEF: peak expiratory flow; FEV1: forced expiratory volume in $1 \mathrm{~s}$; VC: vital capacity; FVC: forced vital capacity.

parameters needed as well as the variability in the different outcomes assessed and epidemiological designs used. Although nine studies satisfied all the requirements for meta-analysis, it was not undertaken as the high heterogeneity would have generated evidence of poor quality (table 3 ). 


\section{Discussion}

This systematic review identified 21 studies assessing the impact of pharmacists' interventions on asthma outcomes in adult patients. A large variety of outcomes were used to demonstrate such impact, including different measures of asthma control, asthma severity, pulmonary function and asthma symptoms.

(C)-RCTs generated similar results to those generated by non-RCTs for most of the outcomes assessed. Nine out of 10 studies assessing asthma control revealed a positive impact after the provision of pharmacists' interventions. For example, all studies reporting changes in the percentage of controlled asthma patients found a positive association between pharmacists' interventions and current asthma control [15, 19, 22, 27]. Similar results were observed in terms of ACQ scores, with all six studies reporting significant improvements for this outcome $[15,19,20,25,26,33]$. Two of the four studies measuring ACT scores found a similar trend [17, 27]. Studies assessing any measure of asthma severity also supported the potential role of pharmacists in asthma management. Pharmacists contributed to a significant reduction in the percentage of severe patients and asthma severity scores in three of the four studies assessing this outcome [14, 21, 23]. Both RCTs and non-RCTs assessing pulmonary function measures failed to demonstrate a significant impact on VC, FVC, FEV1/FVC and FEV1 \% VC. However, improvements were identified in terms of PEF and FEV $1[18,21,23,27,28,30,32]$. Regardless of the study design, pharmacists were found to have a positive impact on different measures of asthma symptoms. For example, seven studies assessing general or nocturnal symptoms as their main outcome reported improvements after the provision of the intervention [16, 18, 21, 22, 29-31]. It is evident that both (C)-RCTs and non-RCTs yielded similar trends, highlighting the potential role of pharmacists in asthma management. However, appropriately designed, conducted and reported (C)-RCTs represent the gold standard in assessing healthcare interventions [35]. Future research aimed at assessing pharmacists' interventions on asthma outcomes should utilise these research designs. Nevertheless, further experimental designs can be considered if a conventional RCT design is not feasible [36]. The use of other research designs would also be of great interest, allowing the assessment of the elements of the interventions. It is worthwhile mentioning that amongst the included studies, many different interventions with several interacting components were tested. This heterogeneity may have impacted the outcomes achieved, and may account for the differences between significant and nonsignificant findings. As the effect of the different intervention elements was not independently assessed in any of the studies, those with the biggest impact on clinical asthma outcomes remain unknown.

Within the 21 included studies, 11 different clinical outcomes and 26 different reporting systems were identified, hindering the synthesis of the evidence available. As in other conditions [37, 38], it is necessary to agree on a core outcome set to be measured and reported in all the studies assessing the impact of health interventions on adult asthma patients. The selection of appropriate outcomes to measure allows the analysis of the effects of different interventions and minimises bias [10]. This provides not only a reliable comparison of results across different studies, but also an evaluation of the consistency of the research findings when translated into clinical practice. The core outcome set for asthma interventions must be established in accordance with updated evidence-based reports, such as the Global Initiative for Asthma (GINA). In its 2015 update, GINA recommends the assessment of asthma control based on two different domains: symptom control (previously known as current clinical control) and future risk of adverse outcomes (including risk factors for exacerbations, fixed airflow limitation and medication side-effects) [3]. This implies that both domains should always be assessed and reported separately. The application of this approach to assess the impact of pharmacists' interventions seems to be feasible. We suggest that studies assessing pharmacists' interventions in clinical trials always report at least symptom control and specific indicators of risk of adverse outcomes as a core outcome set, described as follows. The assessment of symptoms control should be done using validated tools, such as the ACQ and the ACT. These instruments have already been used in several intervention studies with positive results $[15,19,20$, $25-27,33,34]$. In terms of a future risk of adverse outcomes assessment, at least short-acting $\beta_{2}$-agonist use, adherence to inhaled corticosteroids, inhaler technique, exposure to triggers and potentially FEV 1 should be measured and reported. None of the studies included in this review reported all the aforementioned outcomes. This might be explained by the fact that first asthma severity, and then current clinical control, have traditionally been the ultimate outcomes of asthma management.

A meta-analysis of the studies was attempted to estimate the pooled effects of pharmacists' interventions on asthma outcomes. However, some factors impeded its performance, such as the variability on the different outcomes assessed and the reporting systems used, together with lack of statistical parameters. Different guidelines for reporting experimental studies (e.g. CONSORT [39]) have been developed to assist authors in writing manuscripts, and journal editors and peer reviewers in evaluating them for publication. This has undoubtedly helped to increase the quality of data available in the scientific community. However, whilst key statistical parameters are missing or high variability remains common, identifying a real effect of an intervention will not be possible. 
A potential limitation of this systematic review is that some studies undertaken in the early 2000 s assessing the role of pharmacists on asthma management were retrieved in our search strategy, but not included in the analysis [40-42]. These papers included children as well as adults and we reviewed studies which dealt with adults only. Although our query intended to retrieve all published studies evaluating the effect of pharmacists' interventions in clinical asthma outcomes, publication bias may have occurred because no grey literature was included. However, since no papers were retrieved from additional references, it seems that high-sensitivity queries were used. This may also be explained due to the number of databases used, covering most of the published data in our research area. Due to the low number of (C)-RCTs identified, studies with different experimental research designs were included. This impeded performing a risk of bias assessment. However, following AMSTAR recommendations, this was taken into account for the data synthesis and formulation of the conclusions of this review [13]. Moreover, the potential differing quality of the studies included did not affect the main objective of our review.

In conclusion, the evidence of pharmacists' interventions on clinical asthma outcomes in adult patients has been evaluated using heterogeneous outcomes, including different measures of asthma control, asthma severity, pulmonary function and asthma symptoms. Based on the evidence generated by (C)-RCTs, pharmacists' interventions have a positive impact on the percentage of asthma-controlled patients, ACQ scores, asthma severity and asthma symptoms. Inconsistent impact has been found in terms of ACT scores and pulmonary function indicators. Future research evaluating the impact of pharmacists' interventions on clinical asthma outcomes should report using a core outcome set of indicators established for this condition, based on GINA recommendations.

\section{References}

1 World Health Organization. Asthma fact sheet 307, 2013. www.who.int/mediacentre/factsheets/fs307/en/ Date last accessed: January 22, 2015.

2 Braman SS. The global burden of asthma. Chest 2006; 130: 1 Suppl., 4s-12s.

3 Global Initiative for Asthma. Global strategy for asthma management and prevention 2015. http://www.ginasthma. org/documents/4 Date last accessed: April 5, 2015.

4 Haughney J, Price D, Kaplan A, et al. Achieving asthma control in practice: understanding the reasons for poor control. Respir Med 2008; 102: 1681-1693.

5 Horne R, Price D, Cleland J, et al. Can asthma control be improved by understanding the patient's perspective? BMC Pulm Med 2007; 7: 8.

6 Machado M, Bajcar J, Guzzo GC, et al. Sensitivity of patient outcomes to pharmacist interventions. Part I: systematic review and meta-analysis in diabetes management. Ann Pharmacother 2007; 41: 1569-1582.

7 Machado M, Bajcar J, Guzzo GC, et al. Sensitivity of patient outcomes to pharmacist interventions. Part II: Systematic review and meta-analysis in hypertension management. Ann Pharmacother 2007; 41: 1770-1781.

8 Fathima M, Naik-Panvelkar P, Saini B, et al. The role of community pharmacists in screening and subsequent management of chronic respiratory diseases: a systematic review. Pharm Pract (Granada) 2013; 11: 228-245.

9 Benavides S, Rodriguez JC, Maniscalco-Feichtl M. Pharmacist involvement in improving asthma outcomes in various healthcare settings: 1997 to present. Ann Pharmacother 2009; 43: 85-97.

10 Williamson PR, Altman DG, Blazeby JM, et al. Developing core outcome sets for clinical trials: issues to consider. Trials 2012; 13: 132.

11 Gargon E, Williamson PR, Altman DG, et al. The COMET Initiative database: progress and activities from 2011 to 2013. Trials 2014; 15: 279.

12 Liberati A, Altman DG, Tetzlaff J, et al. The PRISMA statement for reporting systematic reviews and meta-analyses of studies that evaluate health care interventions: explanation and elaboration. PLoS Med 2009; 6: e1000100.

13 Shea BJ, Grimshaw JM, Wells GA, et al. Development of AMSTAR: a measurement tool to assess the methodological quality of systematic reviews. BMC Med Res Methodol 2007; 7: 10.

14 Armour C, Bosnic-Anticevich S, Brillant M, et al. Pharmacy Asthma Care Program (PACP) improves outcomes for patients in the community. Thorax 2007; 62: 496-502.

15 Armour CL, Reddel HK, LeMay KS, et al. Feasibility and effectiveness of an evidence-based asthma service in Australian community pharmacies: a pragmatic cluster randomized trial. J Asthma 2013; 50: 302-309.

16 Barbanel D, Eldridge S, Griffiths C. Can a self-management programme delivered by a community pharmacist improve asthma control? A randomised trial. Thorax 2003; 58: 851-854.

17 Bereznicki BJ, Peterson GM, Jackson SL, et al. Pharmacist-initiated general practitioner referral of patients with suboptimal asthma management. Pharm World Sci 2008; 30: 869-875.

18 Petkova E. Evaluation of the impact of a pharmaceutical care program on patients with asthma. Ankara Universitesi Eczacilik Fakultesi Dergisi 2005; 34: 251-262.

19 Garcia-Cardenas V, Sabater-Hernandez D, Kenny P, et al. Effect of a pharmacist intervention on asthma control. A cluster randomised trial. Respir Med 2013; 107: 1346-1355.

20 Giraud V, Allaert FA, Roche N. Inhaler technique and asthma: feasability and acceptability of training by pharmacists. Respir Med 2011; 105: 1815-1822.

21 Mangiapane S, Schulz M, Muhlig S, et al. Community pharmacy-based pharmaceutical care for asthma patients. Ann Pharmacother 2005; 39: 1817-1822.

22 Mehuys E, Van Bortel L, De Bolle L, et al. Effectiveness of pharmacist intervention for asthma control improvement. Eur Respir J 2008; 31: 790-799.

23 Saini B, Krass I, Armour C. Development, implementation, and evaluation of a community pharmacy-based asthma care model. Ann Pharmacother 2004; 38: 1954-1960. 
24 Schulz M, Verheyen F, Muhlig S, et al. Pharmaceutical care services for asthma patients: a controlled intervention study. J Clin Pharmacol 2001; 41: 668-676.

25 Smith L, Bosnic-Anticevich SZ, Mitchell B, et al. Treating asthma with a self-management model of illness behaviour in an Australian community pharmacy setting. Soc Sci Med 2007; 64: 1501-1511.

26 Toumas-Shehata M, Price D, Basheti IA, et al. Exploring the role of quantitative feedback in inhaler technique education: a cluster-randomised, two-arm, parallel-group, repeated-measures study. NPJ Prim Care Respir Med 2014; 24: 14071.

27 Zanghelini F, Filho J, Carvalho A, et al. Impact of pharmaceutical care on lung function in patients with severe asthma. Revista de Ciencias Farmaceuticas Basica e Aplicada 2013; 34: 379-386.

28 Anjan Kumar DS, Adepu R, Parthasarathi G, et al. Impact of community pharmacist provided patient education in asthma patients on treatment outcomes - a study. Indian J Educ Res 2009; 43: 125-133.

29 Abdelhamid E, Awad A, Gismallah A. Evaluation of a hospital pharmacy-based pharmaceutical care services for asthma patients. Pharmacy Practice 2008; 6: 25-32.

30 Narhi U, Airaksinen M, Tanskanen P, et al. Therapeutic outcomes monitoring by community pharmacists for improving clinical outcomes in asthma. J Clin Pharm Ther 2000; 25: 177-183.

31 Odegard PS, Lam A, Chun A, et al. Pharmacist provision of language-appropriate education for Asian patients with asthma. J Am Pharm Assoc (2003) 2004; 44: 472-477.

32 De Tullio PL, Corson ME. Effect of pharmacist counseling on ambulatory patients' use of aerosolized bronchodilators. Am J Hosp Pharm 1987; 44: 1802-1806.

33 Lim AS, Stewart K, Abramson MJ, et al. Multidisciplinary Approach to Management of Maternal Asthma (MAMMA): a randomized controlled trial. Chest 2014; 145: 1046-1054.

34 Young HN, Havican SN, Griesbach S, et al. Patient And phaRmacist Telephonic Encounters (PARTE) in an underserved rural patient population with asthma: results of a pilot study. Telemed J E Health 2012; 18: 427-433.

35 Sedgwick P. What is a non-randomised controlled trial? BMJ 2014; 348: g4115.

36 Craig P, Dieppe P, Macintyre S, et al. Developing and evaluating complex interventions: the new Medical Research Council guidance. BMJ 2008; 337: a1655.

37 Benstoem C, Moza A, Autschbach R, et al. Evaluating outcomes used in cardiothoracic surgery interventional research: a systematic review of reviews to develop a core outcome set. PLoS One 2015; 10: e0122204.

38 Sinha IP, Gallagher R, Williamson PR, et al. Development of a core outcome set for clinical trials in childhood asthma: a survey of clinicians, parents, and young people. Trials 2012; 13: 103

39 Schulz KF, Altman DG, Moher D. CONSORT 2010 statement: updated guidelines for reporting parallel group randomised trials. BMJ 2010; 340: c332.

40 Cordina M, McElnay JC, Hughes CM. Assessment of a community pharmacy-based program for patients with asthma. Pharmacotherapy 2001; 21: 1196-1203.

41 Herborg H, Soendergaard B, Froekjaer B, et al. Improving drug therapy for patients with asthma - part 1: Patient outcomes. J Am Pharm Assoc (Wash) 2001; 41: 539-550.

42 McLean W, Gillis J, Waller R. The BC Community Pharmacy Asthma Study: a study of clinical, economic and holistic outcomes influenced by an asthma care protocol provided by specially trained community pharmacists in British Columbia. Can Respir J 2003; 10: 195-202. 